Spectator rekurriert. Wird Spectator mit Majuskel und kursiv geschrieben, so handelt es sich um die spezifische Schrift: The Spectator (1711-1714). Ähnlich verhält es sich im deutschen Sprachgebrauch, in dem die spezifischen Zeitschriften stets in Kursivschrift gesetzt werden. Da im Deutschen eine Differenzierungsmöglichkeit durch Groß- und Kleinschreibung wegfällt, wird in der vorliegenden Arbeit in undeutlichen Fällen bei nicht kursiver Typografie des Begriffs ,Spectator' präzisiert, ob von der Spectator-Gattung die Rede ist oder vom Mr. Spectator, das heißt der fiktiven Verfassungsinstanz innerhalb des Spectator - also der Zeitschrift.

Obwohl die von den deutschsprachigen Wochenschriften stammende Bezeichnung ,Moralische Wochenschriften“ aufgrund anderer als wöchentlicher Publikationsrhythmen oder unterschiedlicher Auffassungen von Moral auch für die romanischen Wochenschriften nicht vollkommen akkurat ist (cf. Fischer 2014, 41-42), wird in der vorliegenden Arbeit der deutschsprachige Terminus zur Bezeichnung der Gattung benutzt. Schreib- und Lektüreerfahrungen vergangener Arbeiten zu den englisch-, französischund spanischsprachigen Wochenschriften in verschiedenen Metasprachen haben nämlich offenbart, dass trotz der typografischen Unterscheidung der Synoyme (spectateur, espectador) wiederholt Unklarheit darüber aufkommen kann, ob die Zeitschrift, die Verfassungsinstanz oder die Gattung gemeint ist. Darüber hinaus wird, um einer Lektüremonotonie entgegenzuwirken, neben dem Begriffspaar ,Moralische Wochenschriften` gleichbedeutend von den ,Wochenschriften' (ohne Adjektiv), den ,(moralischen) Periodika', den ,(moralischen) Zeitschriften` oder der ,Spectator-Gattung ' die Rede sein.

Welche Periodika nun zur Spectator-Gattung gezählt werden, wird unter den Gelehrten bereits im 18. Jahrhundert polemisch debattiert. Die sogenannte ,SpectatorFrage' dreht sich um

[...] issues concerning the true meaning of being a Spectator, of writing as a Spectator, of persuading as a Spectator. As if there had occurred a process of ,sacralization' of the English text, the value of its followers was measured in direct relation to their faithfulness to the original title, to what was believed to be its original form of teaching [kursiv im Orig.]. (Pallares-Burke 1996, 7)

Da diese Frage bis heute aktuell und für die vorliegende Studie bedeutend ist, werden nunmehr die von Ertler ausgearbeiteten Merkmale der Spectator-Gattung dargestellt.

\title{
1.4 Spectatoriale Gattungsmerkmale
}

Die ungeheure Beliebtheit der Spectator-Blätter im 18. Jahrhundert lässt sich zweifelsohne auf ihre charakteristischen Merkmale zurückführen. In seinem Überblicksartikel zu den Moralischen Wochenschriften nennt Klaus-Dieter Ertler (2012a) sechs prototypische spectatoriale Merkmale, die als Ausgangspunkt für die systematische Beschreibung ihrer ästhetisch-narrativen Formprinzipien dienen und bei der Analyse der spectatorialen Geschlechterkonstruktionen berücksichtigt werden. Zu diesen zählen (1) die periodische Erscheinungsweise und Neuauflagen, (2) die Übersetzungen, Nachahmungen und Adaptationen, (3) das (neuerdings explizit adressierte) weibliche Lesepublikum, (4) die fiktive Herausgeber*innen- und Autor*inneninstanz, (5) die 
Inszenierung von Soziabilität und (6) die Vielfalt der literarischen Formen und Gattungen. Abschließend werden Ertlers Gattungsmerkmale um ein siebentes - jenes der Menschenbeobachtung - erweitert, da es für die moralische Besserung und Selbstdisziplinierung der (bürgerlichen) Gesellschaft ganz zentral ist, dass diese erstens erkennen und zweitens beurteilen lernt, welches Verhalten (nicht) angebracht ist, um darauf angemessen reagieren zu können.

\subsubsection{Periodische Erscheinungsweise und Neuauflagen}

Als erstes (technisch-mediales) Gattungsmerkmal nennt Ertler die periodische Erscheinungsweise und Neuauflagen. Die Moralischen Wochenschriften erscheinen an bestimmten Wochentagen, wobei der Publikationsrhythmus je nach Zeitschrift von täglich bis zweiwöchentlich reicht. Da sie relativ wenig tagespolitische Inhalte transportieren, sind ihre Beiträge eher zeitloser Natur und werden noch Jahre, gar Jahrzehnte später in Buchform und oft in mehreren Bänden herausgegeben. Noch am Ende des 18. Jahrhunderts beschreibt Suzanne Necker (1737-1794) - Mutter von Madame de Staël (1766-1817) - in ihrem posthum erschienenen Werk Nouveaux mélanges extraits des manuscrits de Madame Necker (1801) die Abwechslung jedes SpectatorKapitels als entscheidende Ingredienz für den Erfolg aller Spectator-Periodika:

Chaque chapitre se varierait : l'un serait sur la lecture, un autre sur le goût, un troisième porterait le nom d'un grand homme ou d'une femme célèbre : ce serait encore un jugement sur la correspondance de Voltaire, un parallèle du style de Rousseau et de Thomas ; des idées sur le style en général ; du style épistolaire ; un voyage ; des Gracques et d'autres tragédies ; des pensées détachées sur tel ou tel sujet; lettres à $M$. un tel sur un livre ou sur un événement, sur l'esprit du moment. On réunirait quelques fois deux ou trois lettres en une, pour les rendre plus piquantes ; [...]. (Necker 2013, 257-258)

So ist es nicht verwunderlich, dass die 555 Nummern des Spectator, die zwischen 1. März 1711 und 6. Dezember 1712 täglich (bis auf Sonntag) erscheinen, bereits zwischen 1712 und 1713 in einer ersten Serie von sieben Bänden veröffentlicht werden. 1715 kommt eine zweite Bandserie mit insgesamt 635 Nummern heraus, die um den achten Band (das heißt mit den zwischen 18. Juni und 20. Dezember 1714 veröffentlichten Artikeln) erweitert wird. Weitere Bandauflagen der 635 Spectator-Nummern entstehen über das gesamte Jahrhundert hinweg (1729-1739, 1744, 1765, 1778, 1793 1794) und noch weit darüber hinaus, wobei die Auflagen ab Ende des 18. Jahrhunderts immer häufiger mit historischen Einleitungen, biografischen Notizen, Annotationen etc. versehen werden (cf. Raquejo 1991, 18-19). ${ }^{23}$

Auch Alain Bony $(1999,200)$ nennt als das wahrhaft Neue an Steeles und Addisons Zeitschrift die täglichen Auflagen, die er in Verbindung mit der neuartigen Essayform

23 Scott Black (2008) sieht in den Bandauflagen einen wichtigen Beitrag, mit dem die Schreibund Lesepraxis des Publikums geschult und somit auf die Produktion und Rezeption der Romangattung vorbereitet wird. 
bringt, die sich über den Spectator in England verbreitete. ${ }^{24}$ In den 635 Essays, von denen jeder einzelne einer Ausgabennummer entspricht, vereinen Addison und Steele Merkmale früherer Essay-Schreibender wie John Dunton (1659-1733), Daniel Defoe (1660-1731), Jonathan Swift (1667-1745) oder Michel de Montaigne (1533-1592). Auf diese Weise etablieren sie ihre eigene essayistisch-narrative Ausdrucksweise, in der sie einen persönlichen, dialogorientierten, familiären Stil mit einem satirisch-humorvollen und gleichzeitig moralisierenden Ton an den Tag legen (cf. Boulard Jouslain 2017; Turner 1949, 11-16). Ebenfalls entwickelt Addison in den Spectator-Nummern 411 bis 421 seine eigene ästhetische Theorie, mit der er sich an den Beginn der Ästhetik der Neuzeit stellt (cf. Stolzenberg 2012) und in die Schulbücher und Anthologien des 18. Jahrhunderts Eingang findet (cf. Mack 1994).

\subsection{2 Übersetzungen, Nachahmungen und Adaptationen}

Neben den Neuauflagen werden von den englischen Prototypen ausgehend schon bald nach deren Entstehen Übersetzungen, Nachahmungen und kulturelle Adaptationen angefertigt. ${ }^{25}$ Als , Übersetzungen “ werden weitgehend originalgetreue Übersetzungen in andere Sprachen verstanden; als ,Nachahmungen “ Wochenschriften, in denen die gattungsspezifischen und inhaltlichen Muster der englischsprachigen Prototypen deutlich reproduziert werden, und als ,kulturelle Adaptationen ' Wochenschriften, die ihre Formen und Inhalte an die jeweilige Zielkultur anpassen (cf. Lüsebrink 2016, 147-148).

Unter den drei Prototypen - The Tatler (1709-1711), The Spectator (1711-1712; 1714) und The Guardian (1713) - ist es vor allem der Spectator, der sich in der Welt der Wochenschriften durchsetzen wird. Wolfgang Martens (1968, 23-24) beschreibt die Entwicklungsstufen beginnend mit dem Tatler folgendermaßen: Obwohl dieser sich ,anfangs seiner Gestalt noch nicht sicher“ war, hat er „die Charakteristika der Gattung entwickelt", die der Spectator im Anschluss ,klassisch fixiert“" hat und die mit dem Guardian schließlich ,beglaubigt" wurden.

Ziel der Übersetzungen und Nachahmungen des ursprünglichen Spectator ist es, das Gemeinwohl der Bevölkerung zu fördern, wobei es immer wieder auch zu kulturellen Adaptationen der ursprünglichen Formen und Inhalte kommt. So entwickeln die spanischen Wochenschriften beispielsweise die Erzählform des Briefes weiter (cf. Hobisch 2017), oder es zirkulieren über das Jahrhundert hinweg diverse Mikroerzählungen in den verschiedensten europäischen Periodika (cf. Fuchs et al. 2019). Bereits Zeitgenossen wie Daniel Defoe (1660-1731) und Comte Caylus (1692-1765) nehmen wahr, dass die Spectator-Periodika wie Pilze aus dem Boden schießen, und loben ihre wirkmächtige Vorbildfunktion: „One of the qualities most often attributed to the Spectator was the power to correct vicious, faulty and inappropriate styles of thought and

24 Zur Entwicklung der Essayistik, an deren Anfang neben den englischen Moralischen Wochenschriften auch die Tradition des ,familiar essays' steht, siehe Albert Göschl (2016) Die Logik des essayistischen Gedankens, inbesondere Kapitel 3 zur Genese der Forschungsdiskurse.

25 Ertlers zweites Gattungsmerkmal der Übersetzungen und Adaptationen greift für die Moralischen Wochenschriften zu kurz, weshalb hier zusätzlich ihre Nachahmungen angeführt werden. 
behaviour and to redirect them along reasonable and humanitarian paths [kursiv im Orig.]“ (Pallares-Burke 1996, 2). Jean-Jacques Rousseau (1712-1778), zum Beispiel, rät in Émile ou de l'éducation (1762) der Protagonistin, den Spectator zu lesen, um so die Aufgaben einer ,honnête femme' (einer der Gesellschaft nützlichen bürgerlichen Frau) zu erlernen: „,...] le Spectateur, dont vous [Sophie] aimez la lecture. Étudiez-y les devoirs des honnêtes femmes, et songez que dans deux ans ces devoirs seront les vôtres“ (Rousseau 1762, Livre V, 87). Oft wird das Lesen der englischen Wochenschriften und ihrer Nachfolgerinnen, das als heilsam für die Gesundheit des Geistes angesehen wird, mit dem Schlucken heilender Medizin verglichen (cf. Pallares-Burke 2004, 147). Der Verfasser des Nouveau Spectateur français (1723-1725) sieht seine Aufgabe gar als ,Sittenmediziner‘ (médecin des mœurs):

Les maladies morales sont l'extravagance \& le vice ; l'unique remède propre à les déraciner, c'est la raison : l'industrie d'un Medecin des mœurs consiste, à se rendre agréable à ceux qui sont entre ses mains, à se plier à leur humeur, à s'insinuer dans leur [sic] chimères, pour les emploïer de concert avec les maximes du bon sens à leur propre destruction. (NS1 1,5)

Die Beliebtheit der Spectator-Gattung zeigt sich vor allem an ihrem umgehenden Transfer auf den europäischen Kontinent und ihre über das Jahrhundert hinweg anhaltende Rezeption (siehe Unterkapitel zum Entstehungs- und Verbreitungskontext). Maßgebend wirken die kurz nach Erscheinung des Spectator angefertigten französischsprachigen Übersetzungen, Nachahmungen und Adaptationen nicht nur in den romanischen Raum, sondern über den Umschlagplatz Leipzig bis nach Polen und Russland hinein (cf. Rau 1980, 168). Auffällig ist, dass die moralischen Periodika in vielen weiteren europäischen Sprachen verfasst werden, was darauf zurückzuführen ist, dass im 18. Jahrhundert die meisten Wochenschriftenproduzent*innen von einer Differenz der Völker/Nationen ausgehen. Das heißt, den unterschiedlichen Völkern/Nationen werden bestimmte (Charakter-)Eigenschaften zugeschrieben, die es zu ,bedienen' gilt. Im Vorwort des Verlegers von Bastides Nouveau Spectateur (1758-1760) wird die Notwendigkeit einer Wochenschrift auf Französisch zum Beispiel folgendermaßen legitimiert: „,[U]n Observateur des mœurs Angloises, est, à bien des égards, un moraliste très-étranger en France. Chaque Nation a ses principes, ses usages, son génie qui la caractérisent" (NS2, Avis des Libraires, 4). Aus diesem Grund brauche jede Nation ihre eigene Wochenschrift, denn ,il faut l'offrir à elle-même en spectacle; il faut qu'elle puisse s'appliquer les réflexions qu'elle lit; sans cela elle ne lira que pour son amusement" (NS2, Avis des Libraires, 4-5). Um die Sitten und Verhaltensweisen der französischen Gesellschaft zu bessern, könne demnach nur eine Beobachtungsinstanz französischer Herkunft die Gesellschaft betrachten und ihre Systeme, Handlungen und Intrigen darstellen.

\subsubsection{Weibliches Lesepublikum}

Als geschlechtsspezifische Adaptation der Spectator-Prototypen können ihre feminisierten Titel gewertet werden. Bereits parallel zum Tatler entsteht The Female Tatler 
(1709-1710), ${ }^{26}$ in Frankreich erscheint anonym La Spectatrice (1728-1729) und dreiBig Jahre nach dem Spectator verfasst Eliza Haywood The Female Spectator (17441746), ${ }^{27}$ eine in vielen Ländern Europas nachgeahmte Schrift. Durch die ausdrückliche Adressierung des weiblichen Lesepublikums erfährt dieses innerhalb der gesamten Wochenschriftengattung eine explizite Aufwertung und wird deshalb von Ertler als drittes Gattungsmerkmal genannt.

Insgesamt richten sich die Moralischen Wochenschriften mit dem Ziel, eine dem Gemeinwohl dienliche Grundbildung ${ }^{28}$ für ein breiteres Publikum bereitzustellen, an „, Gelehrte und Ungelehrte zugleich, ' [...] jedoch nicht an die breite Masse oder gar die Unterschicht“"29 (Mlitz 2008, 137-138). Die adressierten Leser*innen stammen vorwiegend aus dem Handel treibenden Bürgertum, das sich von der landbesitzenden Klasse abhebt und durch eine pragmatisch-rationalistische Lebenseinstellung auszeichnet (cf. Stürzer 1984, 25). Die Blätter verfolgen also

26 Die Publikation des Female Tatler wird teilweise einer Frau, Delarivière Manley (16631724) (cf. Melton 2001, 149; Reddy/Gershenson Smith 2002), teilweise einem Mann, Thomas Baker (1680-1749) (cf. Boulard 2000, 456), zugeschrieben und wurde immer wieder in der Forschung diskutiert (cf. Anderson 1931; Smith 1952). Dessen ungeachtet ist weibliche Autorschaft im 18. Jahrhundert eher die Ausnahme als die Regel, da Frauen der Zugang zu höherer Bildung und den Berufen, die viele Autoren unterstützten (Rechtswissenschaft, Medizin, Hochschullehre, bürokratischer Dienst), verwehrt bleibt. Sogar wohlhabende bürgerliche oder aristokratische Frauen, die eine relativ umfangreiche Ausbildung von Familientutor*innen erhalten haben, riskieren soziale Missbilligung, wenn sie sich entscheiden, ihre Werke zu veröffentlichen. Es befinden sich jedoch sehr viele Frauen im Publikum, weshalb es auch immer wieder spezifisch an Frauen gerichtete Zeitschriften gibt. Zwischen 1700 und 1789 erscheinen circa 25 französischsprachige und 85 deutschsprachige Periodika. In England kommen 80 Periodika zwischen 1690 und 1760 heraus (cf. Melton 2001, 148-150). Ebenfalls explizit an ein weibliches Publikum richten sich im 18. und 19. Jahrhundert die sogenannten Damenalmanche, wie der deutsch-französische Almanach des Dames (18011840), der ,insbesondere durch die Valorisierung und Kanonisierung weiblicher Schriftsteller und damit auch als Medium der Wortergreifung von Schriftstellerinnen [hervorsticht]“ (Lüsebrink/Haß 2013, 287).

27 The Female Spectator ist die erste bekannte englische Spectator-Nachahmung aus der Feder einer Frau: Eliza Haywood. Mit ihrer Zeitschrift tritt Haywood in den öffentlichen Diskurs und erhebt sich implizit gegen das restriktive Frauenbild, das ihre männlichen Kollegen propagieren. Inhaltlich orientiert sich die Zeitschrift jedoch ebenfalls an den geschlechtsspezifischen Konventionen der Zeit. Als bekannteste der zahlreichen europäischen Spectator-Imitationen, die sich einer weiblichen fiktiven Herausgeberfigur bedienen, erfährt The Female Spectator zahlreiche Nachahmungen in anderen europäischen Ländern.

28 Zur Entwicklung und zum Bedeutungswandel des Begriffes ,Bildung' aus geschlechtsspezifischer Perspektive siehe Andrea Felbinger (2004) Der Wandel des Bildungsbegriffes unter feministischer Perspektive. Auf den Spuren der Geschlechterbildung.

29 Dezidiert an das breite Volk am Land richten sich den Wochenschriften ähnliche Zeitschriften wie die Bauern- und Dorfzeitschriften (cf. Faulstich 2002, 35-37). 
[...] eine klare Zielgruppenpolitik, in der zunächst die alte Kluft zwischen dem akademischliterarisch versierten Gelehrtenbürgertum in den Funktionsstellen von Staat und Kirche und der erwerbsbürgerlichen, vor allem kaufmännischen Mittelschicht zumindest intentional, im Interesse des ,juste milieu', außer Kraft gesetzt w[i]rd[...] - in der Abwehr des gelehrten Pedantismus wie auch der mit dem Hof assoziierten Galanterie. (Kühlmann 2012, 21)

Die Lektüreempfehlungen, die Zitate und gleichzeitigen Übersetzungen antiker Satiren und moralistischer Werke sowie die Maximen, die mit Zitaten von bekannten zeitgenössischen Persönlichkeiten untermauert werden, sieht Kühlmann als Indiz dafür. Durch einen Blick in die im Spectator erschienenen Leser*innenbriefe identifiziert auch Fritz Rau die neuen bürgerlichen Schichten als Zielgruppe der Periodika, zu denen ,modische Damen, Geschäftsleute, Geistliche, Spieler, Dienerschaften, Liebhaber, Schüler und bekümmerte Eltern“ (Rau 1980, 71) zählen. Die abgedruckten Annoncen lassen ebenfalls Rückschlüsse auf die Herkunft des Publikums aus unterschiedlichen sozialen Milieus und politischen Lagern zu (cf. ibid., 73). Durch den großen Einfluss, den die Wochenschriftenproduzent*innen auf ihr Publikum ausüben, betrachtet er die Periodika zudem als eine ,unterhaltsam belehrende[...] und spöttisch erziehende[...] aufklärerische[...] Volksliteratur" (ibid., 71). Andrea Mlitz $(2008,138)$ erwähnt überdies drei Voraussetzungen, mit denen die Wochenschriftenleser*innen ausgestattet sein müssen: Erstens müssen sie über die nötigen finanziellen Mittel verfügen, um die Periodika abonnieren oder kaufen zu können; zweitens muss der Wunsch nach Bildung und drittens die notwendige Zeit vorhanden sein. In Bezug auf das weibliche Lesepublikum beschränken sich diese erforderlichen Faktoren somit auf Leserinnen, die dem gehobeneren Bürgertum oder Adel angehören.

Das weibliche Lesepublikum wird in den moralischen Periodika aber nicht nur dezidiert angesprochen, sondern es wird ihm auch ermöglicht, selbst Beiträge zu verfassen oder ganze Zeitschriften herauszugeben, wodurch diese einen wesentlichen Beitrag zur Wahrnehmung von Frauen im öffentlichen Raum leisten: ,[E]stas voces de mujeres, [...], juegan un papel decisivo en el proceso de acostumbrar a los lectores a la escritura femenina así como en institucionalizarla como presencia pública“"(Urzainqui 2006, 136). Indem die herausgebende Instanz feminisiert wird, wird das englische Modell weiterentwickelt und auch im romanischen Raum für das weibliche Zielpublikum adaptiert. Hier entstehen neue Zeitschriften wie La Spectatrice (1728-1729), La Spectatrice danoise (1748-1750), La Bigarure (1749-1753), La Pensadora gaditana (1763-1764) oder La Pensatriz salmantina (1777), wobei hinter der Produktion der ,weiblichen Wochenschriften' allerdings häufig Männer stehen.

Wie ihre englischen Vorgängerinnen sind die meisten Periodika aus dem romanischen Raum von einer anonymen und fiktiven Verfassungsinstanz geschrieben, die, wie Mr. Spectator, als dirigierende Stimme der Zeitschrift fungiert. Durch den fehlenden Eigennamen entbehren die Verfassungsinstanzen jeglicher Individualität (cf. Van Delft 2005b, 1) und können als (vermeintlich) neutrale Beobachterinnen fungieren, die ihre unmittelbare soziokulturelle Lebenswelt aus dem Hintergrund heraus studieren und ihre Beobachtungen niederschreiben, ohne selbst in nennenswertem Maße als handelnde Person aufzutreten. Aus einer scheinbaren Selbstverständlichkeit heraus wird bereits beim Spectator im Englischen trotz des ,neutralen`Z Zeitschriftentitels - die englische Bezeichnung ,spectator' sagt im Grunde nichts über das Geschlecht der beobachtenden Person aus - von einem männlichen Beobachter ausgegangen, zumal 
Frauen zur damaligen Zeit üblicherweise nicht schriftstellerisch, journalistisch oder wissenschaftlich tätig waren. Doch erst durch den Zusatz ,Mister ${ }^{`}$ erhält die Verfassungsinstanz des Spectator tatsächlich ihr männliches Geschlecht mit all den Konnotationen, die damit einhergehen. Eine dezidierte Genuszuweisung im Titel erhalten nur die ,weiblichen' englischsprachigen Periodika mit der adjektivischen Beifügung ,female', wie der Female Tatler oder der Female Spectator. In den romanischen Sprachen deutet bereits das grammatische Geschlecht im Titel - Spectateur versus Spectatrice; Pensador versus Pensadora - auf das biologische Geschlecht der Verfassungsinstanz hin. In allen Fällen wird schließlich durch den Wochenschriftentitel der Erwartungshorizont des Publikums nicht nur in Bezug auf den Inhalt der Zeitschrift, sondern auch in Bezug auf ihre Verfassungsinstanz aktiviert.

\subsubsection{Fiktive Herausgeber*innen- und Autor*inneninstanz}

Damit wird das vierte und bemerkenswerteste Gattungsmerkmal der Moralischen Wochenschriften angesprochen: eine fiktive Instanz, die sich als reale/r Herausgeber*in und/oder Autor*in ausgibt. Ertler geht zwar nicht näher auf den Unterschied zwischen Herausgeber*in und Autor*in ein, erläutert jedoch in früheren Publikationen (cf. Ertler 2003a, 26-28; Ertler/Köhldorfer 2010, 11-12, 17-19) den edukativen und demokratisch-säkularisierenden Nutzen dieser Anonymisierungs- und Fiktionalisierungsstrategie:

Die Innovation lag in der Einführung einer anonymen fiktiven Autor- und Herausgeberinstanz, die sich für die Kritik der Sitten besonders eignete, weil sie zum einen einen hohen Grad an Objektivität bei der Beobachtung, Beschreibung und Bewertung der zeitgenössischen Gesellschaft und ihrer Laster bot und diese Kritik zum anderen in ein unterhaltsames Ambiente einbettete. Steele griff bei dieser ersten Nummer auf die bereits aus Jonathan Swifts bekannte Figur Isaac Bickerstaff zurück, wodurch es sich bei diesem ersten Beobachter der zeitgenössischen Gesellschaft um eine in England, insbesondere in London bereits gut bekannte fiktive - und daher gewissermaßen ,vertrauenswürdige ‘ - Figur handelte. Hinter dem Pseudonym Bickerstaff baute Steele eine Rahmenfiktion auf und beobachtete aus dieser Position die Londoner Gesellschaft, die im merkantilen Bereich besondere Aktivität aufwies. (Ertler/Köhldorfer 2010, 11-12)

Im umfassenden Kommunikationssystemmodell, das Michaela Fischer (2014, 81-83) für die französischen Wochenschriften aufstellt, geht sie dezidiert auf die verschwimmenden Grenzen zwischen realer und fiktiver Herausgeber*innen- und Autor*inneninstanz sowie zwischen den beiden Instanzen selbst ein, die mitunter sogar zusammenfallen können. Mit Herausgeber*inneninstanz meint Fischer einerseits die das Periodikum real verlegende Person (französisch ,libraire'), „de[r]en Name am Titelblatt steht[,] und andererseits de[n] vermeintlich fiktive[n] Libraire, der sich in Paratexten an die Käufer/Leser wendet“" (ibid., 82). Schwieriger gestaltet sich die Unterscheidung zwischen realer und fiktiver Autor*inneninstanz, da sich der/die reale Autor*in als Zwischeninstanz an der Textgrenze (also zwischen textexterner und -interner Ebene) befindet und (heute wie damals) nicht festzustellen ist, inwieweit der/die reale Autor*in tatsächlich am Inhalt der Periodika beteiligt war. Auch die Übersetzer*innen von Wochenschriften siedelt Fischer an derselben Stelle als Zwischeninstanz an, da 
diese dieselbe Vermittlungsposition wie die Autor*innen einnehmen. Unter dem/der fiktiven Autor*in versteht Fischer nun diejenige Instanz, die sich als verfassende Figur der Zeitschrift mitunter bereits im Titel vorstellt, wobei „,[d]ie Ausprägung des fiktiven Autors als Verfasserfigur [...] sich vor allem dadurch [äußert], dass diese[r] explizit in den Text eingreift und oftmals bereits die textexterne Ebene ,mitbestimmt', da die Verfasserfigur meist Teil des Titels ist" (ibid., 83).

Nun ist die Abgrenzung der Instanzen der Herausgeber*innen und Autor*innen (sowie der Verfasser*innen) für alle Spectator-Forscher*innen aufgrund der komplexen Kommunikationsstruktur der Wochenschriften und ihrer variablen Beschaffenheit nur in eingeschränktem Maße eindeutig möglich und die einzelnen Instanzenfunktionen (als Herausgeber*in, Autor*in oder Verfasser*in) können sich zudem auch immer wieder überschneiden. Daher führt Fischer den Begriff der Produzent*innenseite ein, um damit eine neutrale Überkategorie zu schaffen. Wie bereits im Vorfeld begonnen, wird auch im weiteren Verlauf der Studie durchgängig neutral ,Produzent' oder ,Produzentin ' verwendet und dort, wo dies eindeutig möglich und notwendig ist, eine Konkretisierung vorgenommen. Diese Vorgangsweise ist insofern gerechtfertigt, als es in dieser Arbeit nicht von Belang sein wird, ob die spectatorialen Geschlechterdiskurse von einer realen oder fiktiven Instanz verfasst worden sind, da davon ausgegangen wird, dass es sich bei den Zeitschriftendiskursen um kulturell und gesellschaftlich vorherrschende Diskurse handelt.

Wozu aber dient dieses Verwirrspiel und was bezwecken die Produzent*innen der Wochenschriften damit? Die Maskierungen ${ }^{30}$ der drei Instanzen (Herausgeber*in, Autor*in, Verfasser*in) unter Rekurs auf Pseudonyme und Asteronyme ist aus mehreren Gründen für die Verbreitung der Gattung von Nutzen: Erstens wird die Zensur daran gehindert, die wahren Autor*innen zu sanktionieren, die - nebenbei bemerkt - im 18. Jahrhundert noch kein Eigentumsrecht über ihre Texte besitzen, was dazu führt, dass fremde Gedanken immer wieder ohne Verweis auf deren Herkunft übernommen beziehungsweise abgeschrieben werden. ${ }^{31}$ Zweitens streben die Produzent*innen

30 In Frankreich gehen der maskierte Journalismus und der erzählende Charakter der Moralischen Wochenschriften mit und vor allem nach der Französischen Revolution verloren. So beobachtet Hélène Boons (2020) in ihrer Analyse von Jacques-Vincent Delacroix' Wochenschriften, dass der Journalist Delacroix in seinen späteren Wochenschriften mehr und mehr unter seinem eigenen (Klar-)Namen schreibt und immer öfter seine persönlichen Erinnerungen in das Geschriebene einfließen lässt.

31 Das erste Copyright-Gesetz in Europa verabschiedet England 1710 mit dem Statute of Anne, in dem das Recht des Autors/der Autorin an seinem/ihrem Werk formuliert ist. In Dänemark verbietet eine Anordnung von 1741 jeder Person das Kopieren oder Verkaufen von Büchern oder Manuskripten, an denen eine andere Person aus Kopenhagen bereits Rechte erworben und erhebliche Kosten dafür auf sich genommen hat. In Frankreich wird das Recht auf geistiges Eigentum (droit d'auteur) im Zuge der Gesetzgebungen der Französischen Revolution erlassen. Erst gegen Ende des 19. Jahrhunderts führen alle europäischen Länder eine Gesetzgebung zum Schutz der Rechte der Autor*innen ein: Spanien im Jahr 1834, Österreich 1846, Dänemark 1857, Portugal 1867, Schweden 1867, Deutschland 1870 und Norwegen 1876. Diese länderspezifischen Gesetzgebungen münden 1886 in den ersten internationalen 
durch das bewusste Auslassen ihrer Namen, ihres Alters, ihres Familienstandes usw. ${ }^{32}$ danach, die Vorurteile der bürgerlichen Leser*innen, die aufgrund dieser Auskünfte über die Produzentin oder den Produzenten entstehen könnten, schon im Vorfeld auszuschalten, damit die Lesenden die Argumente und Inhalte der unterschiedlichsten Beiträge möglichst unvoreingenommen aufnehmen können. Der Versuch, ,vorurteilsfreie“ Leser*innen hervorzubringen, funktioniert allerdings nur bedingt, da bereits viele Periodika-Titel auf die (fiktionalisierte) Persönlichkeit oder Gesinnung der Verfassungsinstanz schließen lassen. Wie sich im Zuge der Analyse zeigen wird, erscheint auch jegliche Vorurteilsfreiheit ausgeschaltet, sobald sich im Titel das, Geschlecht der Wochenschrift' ändert und diese nicht mehr auf eine implizit männliche, sondern eine weibliche Verfassungsinstanz verweist. Generell gilt also, dass die Moralischen Wochenschriften doch „,nicht [ganz] sachlich, sondern persönlich und individuell gefasst [sind], und zwar stets unter Einführung einer Person [i. e. Verfasserin], welche die innere Kohärenz des Organs im Sinne einer fiktiven Verfasserschaft verkörpert[...]. Damit [ist] zugleich eine je eigene Perspektive festgelegt“" (Wilke 2008, 103). In den französischsprachigen Periodika ist dies zum Beispiel bei Le Misantrope, Le Censeur oder L'Indigent philosophe der Fall. Und auch bei den spanischen Wochenschriften befindet sich die fiktive Verfassungsinstanz bereits in den Zeitschriftentiteln von El Pensador, El Murmurador, El Censor, El Observador oder El Apologista:

La lectura de los textos evidencia que esta elección de título no es gratuita, sino que sus autores, hurtando su personalidad privada, se enmascaran tras la figura que les ofrece más posibilidades de proyectar sus objetivos críticos - siempre variaciones de una invencible actitud de observación y de crítica - y con la que tratan de acomodarse en su desarrollo sosteniéndola de manera congruente. (Urzainqui 1995, 195)

Von diesen maskierten Verfassungsinstanzen, die mitunter einen sehr subjektiven Erzählstil in Ich-Form - wie im Brief oder Tagebuch - führen, fühlen sich die Leser*innen direkter angesprochen: „Der familiäre, persönliche Ton, die unmittelbare vertrauliche Wendung an den Leser, das leichte, scherzhafte Element ist nur in einem fiktiven Sprechen möglich, ohne aufdringlich oder abgeschmackt wirken zu müssen“ (Martens 1968, 31). Ferner erleichtert die Fiktionalisierung und gleichzeitige Anonymisierung der Verfasser*innen (und weiterer beitragender anonymer Produzent*innen innerhalb einer Zeitschrift), die Gesellschaft kritisch zu kommentieren und zu hinterfragen sowie einen normsetzenden „Moralkodex [...], der dem bürgerlichen Interessenbereich entgegenk[ommt]“ (Ertler 2012a, §15), zu erstellen, ohne dass dafür eine reale Person zur Rechenschaft gezogen werden kann:

Vertrag zum Schutz der Urheber*innen, die Berner Übereinkunft zum Schutz von Werken der Literatur und Kunst (cf. Pila/Torremans 2016, 13-14).

32 Für die Wochenschriften, die (zumindest vorgeblich) von Frauen verfasst worden sind, lässt sich diese Beobachtung nicht bestätigen (siehe Kapitel 3), da die Produzentinnen sehr wohl auf ihr (ungefähres) Alter wie ihren Familienstand eingehen und diese Angaben zum Diskussionsthema machen. 
In a sense, $[\ldots]$ all the $[\ldots]$ spectators $[\ldots]$ play a game and use a mask in order to denounce the games and masks of society. It is the use of evil against itself, the 'transformation of sickness into remedy' described by Starobinski with reference to Rousseau's strategy of cure, but equally applicable to the spectator genre and its reforming aim. Wearing the mask of privileged spectators who claimed to have a wide, detached and exemplary view of the world, they presented themselves as mediators between the world and the readers, offering them not the spectacle of the world itself, but a view of themselves watching it. (Pallares-Burke 1994, 428-429)

Die Vertrauen einflößende Ich-Erzählinstanz und ihre spielerische Selbstdarstellung, die das Publikum über ihre Identität und Authentizität im Ungewissen lässt, sind genuin literarische Verfahren, die in den anonym erscheinenden Periodika ihre größte Entfaltung erlangen. Für die verfassende Instanz ist dieses Schwanken zwischen dem Spektrum der Fiktionalität und Authentizität deshalb sinnvoll, weil sie sich dadurch zum einen , ,...] als moralische Instanz legitimieren und - in Beobachterposition - auBerhalb der Gesellschaft situieren“ (Gronemann 2013, 149) kann, um ihre „didaktische Funktion zu untermauern und nicht selbst durch mögliches Fehlverhalten ins Fadenkreuz der Kritik zu geraten“ (ibid.). Zum anderen ermöglichen ihr die oszillierenden Standpunkte, das Publikum ,nicht nur zu belehren, sondern auf Augenhöhe mit dem Leser auch zu überzeugen und vorbildhafte Wirkung zu erzielen“ (ibid.), was sie durch ihren persönlichen Kommunikationsstil und den mitunter privaten Ton erreicht, durch den sie ein gewisses Vertrauen zum Publikum herzustellen vermag.

\subsubsection{Inszenierung von Soziabilität}

Über die genannten Aspekte hinaus begeistert das Publikum die gespielte, aber auch echte Inszenierung von Soziabilität, die Ertler als fünfte Besonderheit nennt und womit er eine neue Rolle des Publikums meint - und zwar die Möglichkeit der Leser*innen, sich aktiv in den Entstehungsprozess der Periodika einbringen zu können und dadurch soziale Beziehungen untereinander aufzubauen. Um eine möglichst authentische Vielstimmigkeit zu generieren, rufen die (fiktiven) Produzent*innen dazu auf, Briefe zu verfassen, wodurch die Leser*innen nicht nur selbst zu Mitproduzent*innen der Zeitschriften werden, sondern auch zu Helfershelfer*innen ihrer Aufklärungsabsicht mutieren (cf. Pallares-Burke 2004, 149). Denn die eingesendeten Briefe dienen einerseits als Modelle für die formale Gestaltung von Briefen, andererseits servieren sie verhaltensnormative Beispiele für eine tugendhafte bürgerliche Lebensführung. ${ }^{33}$

Die englischen Prototypen folgen mit ihrer Leser*innenkorrespondenz dem Modell von John Dunton (1659-1733), der bereits in seinem Athenian Mercury (1691-1697) anonyme Briefe abdruckt. Als wahre Pioniere im Hinblick auf die Korrespondenz mit

$33 \mathrm{Zu}$ den unterschiedlichen Brieftypen aus der Feder fiktiver oder realer Leser*innen und zur Funktion der Briefe in den spanischsprachigen Wochenschriften siehe Hobisch (2017) La forma epistolar en los espectadores españoles. Características y tipología de las cartas. Zum Brief als Mittel der Herstellung von Soziabilität im Briefroman Cartas marruecas (1772) siehe Gronemann (2017) „Del lujo ostentoso a la ética del hombre sociable: ocio y sociabilidad en las Cartas marruecas de Cadalso“. 
dem Publikum nennt Donald F. Bond jedoch Peter Motteux' (1663-1718) Gentleman's Journal (1692-1694) und die Supplemente von Daniel Defoes Review (1704-1713) sowie das noch frühere französische Vorbild des Mercure Galant (1672) (cf. Bond 1965, Vol. I, xxxvii).

Die Themen der Leser*innenbriefe im Tatler spiegeln seine breiten Inhalte wider: „Bickerstaff's correspondents gave their opinions or asked questions concerning anything or everything which he dealt with: love, courtship, and marriage; manners and morals and their reform, politics, education, the theater, and then on into various byways“" (Bond 1971, 136).

Im Spectator werden hingegen politische Polemiken ganz bewusst ausgespart und nur Publikumsmeinungen zu Mode, Geschmack, Verhalten oder Geschlechterbeziehungen berücksichtigt. Durch die anonymen Beiträge und die unpersönlichen Diskussionen erhalten diese privaten Angelegenheiten öffentliche Relevanz, womit den Meinungen der Diskussionsteilnehmer*innen eine Allgemeingültigkeit zugestanden wird, die zuvor nur dem Staat und der Kirche vorbehalten war. Die Moralischen Wochenschriften werden somit zur Stimme der Zivilgesellschaft (cf. Warner 2002, 70).

Darüber hinaus kann mit der Inklusion von Leser*innenbriefen der im 18. Jahrhundert hochgehaltene Authentizitätstopos bedient werden. ${ }^{34}$ Als eines der ersten Massenmedien ermöglicht die Spectator-Gattung also eine Many-to-many-Kommunikation, ${ }^{35}$ wie sie aus den sozialen Medien bekannt ist. An den Anfängen dieser heute selbstverständlichen Kommunikationstradition steht der Spectator, der die Darstellung seiner eigenen Zirkulation perfektioniert und darauf achtet, dass öffentliche Diskurse zirkulieren und nicht nur in eine Richtung ausgestrahlt werden (cf. Warner 2002, 71). Durch die Aufnahme von Publikumsreaktionen in die Periodika, auf welche die Lesenden ihrerseits wiederum reagieren können, kann also schon lange vor dem Social-MediaZeitalter jede Leserin und jeder Leser vom Empfangenden zum Sendenden werden. Somit entsteht innerhalb des Zeitschriftenmediums ein öffentlicher Kommunikationsraum von gleichrangigen Mitgliedern mit einer - wenn mitunter auch nur fingierten mehrdimensionalen Kommunikationssituation, die von einem (fiktiven) Produzenten oder einer (fiktiven) Produzentin gestaltet und geleitet wird.

Durch die Partizipation von Leser*innen in Form von (teilweise fingierten) Leser*innenbriefen und die explizite Inklusion der Adressat*innen können die

34 Zur Entwicklung des Authentizitätsbegriffs siehe Knaller (2007) Ein Wort aus der Fremde. Geschichte und Theorie des Begriffs Authentizität.

35 In der Regel zeichnen sich Massenmedien durch eine One-to-many-Kommunikation aus, bei der eine bestimmte Nachricht in einer linearen Kommunikation von einem/einer Sender*in ausgehend an ein möglichst großes Publikum vermittelt wird. Das ist zum Beispiel im Fernsehen oder im Internet beim Versenden eines Newsletters bzw. beim Schreiben eines Blogs der Fall.

In der Many-to-many-Kommunikation dagegen kann jede/r Konsument*in auch zum/zur Produzent*in von Nachrichten werden. Solche interaktiven Kommunikationsplattformen finden sich im Internet unter dem Schlagwort ,Social Media', zu denen soziale Netzwerke (Facebook, Twitter etc.), Medienplattformen (YouTube, Wordpress, Flickr etc.), Businessnetzwerke (Xing, LinkedIn etc.), Empfehlungsplattformen (wie Yelp, Delicious, Diigo etc.) und verschiedene Services im World Wide Web zählen (cf. Mergel et al. 2013, 24-28). 
Wochenschriften als interaktive Texte angesehen werden, die auf dem Papier durch das Einbringen verschiedenartiger, oft konträrer Standpunkte eine gewisse Atmosphäre offener Dialogizität herstellen (cf. Kühlmann 2012, 22-23). ${ }^{36}$ So wird der Spectator als polyfoner Text beschrieben, der, wie die Theaterbühne, zum ludischen Treffpunkt unterschiedlichster Meinungen wird:

The Spectator has been rightfully described as a 'polyphonic text' which performed 'a kind of dialogue' between different voices and subjects. [...] In fact, like the stage, the periodical acted as a meeting-point where the voices of a Locke or a Pope, for instance, could be heard together with those of common men and women, genuine and fictitious, who spoke about their lives and problems. The various voices which were brought together in dialogue and confrontation catered for many tastes and world-views. The contradictions and ambiguities which the text embodied guaranteed its popularity rather than hindering it. But if the different admirers and followers of the Spectator might have disagreed about the message or the doctrine being taught, they all seem to have approved of the play-element, the theatrical aspect of the educational strategy [kursiv im Orig.]. (Pallares-Burke 1996, 12)

Durch eine Vielzahl an Produzent*innenfiguren, deren Beiträge sich wie in einem mündlichen Dialog ständig unterbrechen und überlagern, halten die Moralischen Wochenschriften für die Lesenden eine neue innovative Identifikationsmöglichkeit bereit, die bis dato in dieser Form nicht zur Verfügung stand. Die Nachahmung mündlicher Kommunikation stellt für Alain Bony (1982) einen Erfolgsgaranten für die Beliebtheit der Gattung dar:

L'essai périodique n'est pas devenu un lieu privilégié d'interlocution littéraire pour s'être fait le prolongement de la conversation des salons et des cafés, mais pour en avoir mimé la structure sur un mode fictionnel, au prix d'une rupture qui est la condition de son pouvoir d'instauration; d'un renoncement au face à face au profit d'une relation symbolique, d'un détournement de tout le discours mondain vers un effet de parole [kursiv im Orig.]. (Bony $1982,341)^{37}$

Obwohl dieser Kommunikationsraum für eine breite Öffentlichkeit offen zu stehen scheint, muss erneut erwähnt werden, dass er ein relativ geschlossener Sozialraum bleibt, da er seine Teilnehmer*innen durch seinen Habitus, die Themenwahl, die Referenzen oder die Verständigungsformen (vor-)selektiert: „It appears to be open to indefinite strangers, but in fact selects participants by criteria of shared social space (though not necessarily territorial space), habitus, topical concerns, intergeneric references, and circulating intelligible forms (including idiolects or speech genres)“

36 Wie Sven Engesser (2013) festhält, bildet sich der Leser*innenbrief im 18. Jahrhundert „als Plattform des Partizipativen Journalismus heraus“ (55).

37 „The periodical essay did not become a privileged locus of literary interlocution for being the mere extension of conversations in coffee houses, but for miming their structure in a fictional mode, at the cost of a rupture which is the condition of its innovative power; of renouncing face to face communication in favor of a symbolic relationship; and of reorienting all worldly conversation to produce a speech effect [kursiv im Orig.]“" (Bony 1999, 236). 
(Warner 2002, 75). Bereits der editoriale Club des Spectator zeichnet sich dadurch aus, dass er erstens nur Männer inszeniert, die zweitens jeweils einen anderen sozialen Typus verkörpern. ${ }^{38}$

\subsubsection{Vielfalt der literarischen Formen und Gattungen}

Als letztes konstituierendes Merkmal der Moralischen Wochenschriften erwähnt Ertler die Vielfalt der literarischen Formen und Gattungen, die den Lesenden ein breites Lektüreerlebnis verschaffen (cf. Ertler 2012a, §17). Der Formen- und Gattungsreichtum ${ }^{39}$ umfasst neben den bereits erwähnten Leser*innenbriefen auch Traumerzählungen, Fabeln, Allegorien, Gedichte, Utopien, Charakterskizzen bis hin zu satirischen Darstellungen. Sogar Lektüreempfehlungen, Preisausschreiben und Denksportaufgaben erscheinen in einigen Periodika. Hinter dem Konstruktionsprinzip der Einbindung heterogener Textsorten, die nicht immer klar voneinander abgrenzbar sind, steht das Ziel, dem Publikum in ,moralistisch-plaudernde[m] Ton“ (Rau 1980, 353) zu tugendhaftem Verhalten und feinem Geschmack ${ }^{40}$ zu verhelfen. Zu diesem Zweck bauen die Wochenschriftenproduzent*innen auf eine „strukturelle Abwechslung“ (Maar 1995, 12) beziehungsweise ,variierende Wiederholung“ (Jüttner 2011, 62), bei der derselbe Gegenstand immer wieder, aber mit unterschiedlichen formal-ästhetischen Mitteln und Beispielen, aufgegriffen wird. Als Vorbild für die moralisierend-unterhaltsame Art der Aufbereitung dient das Horaz'sche Diktum des ,prodesse et delectare', wonach ein Text in gleichem Maße belehrend wie unterhaltend auf die Leser*innen wirken solle (cf. Ertler 2012a, §20). Diese Ausgewogenheit von Didaktik und Vergnügen erachtet Fritz Rau sogar als wichtigste „Bedingung für [die] Qualität, [den jahrzehntelangen] Fortbestand und [die nachhaltige] Wirkung“ (Rau 1980, 354) der Periodika, die ohne diese Ausprägung dem raschen Untergang geweiht sind. Mit einer nicht allzu poetischen oder wissenschaftstheoretischen und eher prosaischen Ausdrucksweise, die ebenso profane Angelegenheiten wie das Stillen eines Säuglings thematisiert, wird eine moralische Erziehung propagiert, die zur Sittenverbesserung und zum eigenständigen Gebrauch des Verstandes führen soll. ${ }^{41}$ Angestrebt wird eine Abwendung von „religiösen, biblisch fundierten und kirchlich überwachten Weltbilder[n]“ (Kühlmann 2012, 23) und ein Hinführen der Leser*innen zu einer „,naturrechtlich abgeleitete[n]

38 Zur Beschreibung und Funktion der editorialen Clubs und der englischen Kaffeehauskultur als neue öffentliche Sphäre siehe Bony (1999, 99-110, 241-261), Calhoun (2012) oder Cowan (2004).

39 Fischer (2014, 33-34, 215) spricht von, Gattungshybridität‘.

40 Zur Entwicklung des Geschmacksbegriffs als ästhetische Kategorie, die ausgehend von den Schriften des Spaniers Baltasar Gracián (1601-1658) Ende des 17. Jahrhunderts und im 18. Jahrhundert in ganz Europa zum Mittelpunkt einer neuen Geisteshaltung mutiert, siehe Schümmer (1955).

41 Ähnlich formuliert Ulrike Eisenhut (2011) als didaktisches Ziel der Moralischen Wochenschriften die „Verbreitung von Tugend und Moral unter der Leserschaft“ (99), und zwar, indem Inhalte aus verschiedensten Wissensgebieten „im praktischen Alltag der Adressaten verortet“ (100) und ,im angenehmen Plauderton und in Form von Essais“ (99) wiedergegeben werden. 
und logisch deduzierte[n] [...] Pflichtenlehre“ (ibid., 24), wobei die Maxime gilt: „,Ein vernünftiger Mensch muß als freyes Wesen die Tugend ausüben'. Tugend ist demnach ohne staatliche Intervention Produkt eines freien Selbstzwanges unter literarischer Anleitung“ (ibid., 24-25).

Die Vielfalt literarischer Formen und Gattungen in den Zeitschriften basiert wesentlich auf der Integration von unzähligen kürzeren Erzählungen oder Mikroerzählungen, mit denen die moralisierenden Inhalte narrativ vermittelt werden. Dabei macht es für die Leser*innen der moralischen Periodika keinen großen Unterschied, ob die Erzählungen faktualer oder fiktionaler Natur sind, was sich darauf zurückführen lässt, dass Menschen im Laufe der Evolution gelernt haben, mithilfe von Erzählungen zu lernen und zu überleben: „Through storytelling, vicarious knowledge, even guarded knowledge, is used to help our species survive. We learn, regardless of whether the story being told is 'truth' or 'fiction'“ (Le Hunte/Golembiewski 2014, 73). ${ }^{42}$

\title{
1.4.7 Menschenbeobachtung
}

Die von Klaus-Dieter Ertler (2012a) genannten sechs medienspezifischen Gattungsmerkmale der Periodika werden an dieser Stelle um das siebte konstitutive Element der Technik der Beobachtung erweitert, die über die Vertrauen weckenden (fiktiven) Verfasser*innen an die Leser*innen weitergegeben wird:

\begin{abstract}
As privileged and impartial eyes observing the theatre of the world from a first-class seat in the auditorium, the editorial personae modelled on Mr Spectator assume the role of educators of their respective publics, encouraging them to follow their example and to widen and deepen their view of the world and humankind - that is, to become unbiased spectators themselves [Hervorhebung der Verfasserin]. (Pallares-Burke 1994, 413)
\end{abstract}

Über die Lektüre der Moralischen Wochenschriften lernen die Leser*innen zum einen ihre Lebenswelt und zum anderen sich selbst zu beobachten, wobei vorranging die unmittelbare soziokulturelle Lebenswelt, also die sich konstituierende bürgerliche Gesellschaft in Abgrenzung zu anderen Schichten, im Fokus der Betrachtung steht. Die Umdeutung des Lernbegriffs, der Lernen immer weniger als Tradierung von antikem Wissen versteht und immer mehr mit der Anhäufung von Wissen durch Experimente und Beobachtung der Welt in Verbindung bringt, macht dies möglich (cf. Kenklies 2015, 164). Das Ziel, das mit der intendierten Weitergabe der Beobachtungsfähigkeit in den Spectator-Schriften verfolgt wird, ist jenes, den Blick des Publikums auf seine Lebenswelt und die Menschen zu schulen, damit dieses am Ende selbst die Tugenden und Laster seiner Mitmenschen und seiner selbst wahrnehmen und korrigieren kann von vorurteilsfrei (unbiased) kann jedoch keine Rede sein. Diese neue soziale Praxis des Beobachtens steht im engen Zusammenhang mit der Praxis des Beurteilens, die ebenfalls durch die Lektüre der moralischen Zeitschriften trainiert wird. ${ }^{43}$

42 Auf diesen Punkt wird in Kapitel 2 näher eingegangen.

43 Ein ästhetisches Urteilsvermögen, das heißt ein Urteilsvermögen durch die sinnliche Erfahrung des Sehens (griechisch ,aisthesis': Wahrnehmung, Empfindung, Gefühl), propagiert 
Ein Mangel an Seh- und Urteilsfähigkeit der Individuen des 18. Jahrhunderts lässt sich auf die kirchlichen und staatlichen Autoritäten zurückführen, die damals als unhinterfragte Normsetzungsinstanzen gelten und somit eine öffentliche Meinung obsolet machen. Über die Vermittlung der Praxis des Beobachtens, das ein empirisches Verfahren darstellt, ${ }^{44}$ leiten die Wochenschriftenproduzent*innen ihre Leser*innen zum eigenständigen kritischen Denken an. Sie zeigen ihnen, wie sie sich ihres eigenen Verstandes bedienen können, um den Zustand ihrer „selbstverschuldeten Unmündigkeit" (Kant 1975, 9) zu überwinden und Wissen über die Welt zu erlangen. Der Fokus auf den beobachtenden Blick, der schließlich namensgebend für die Gattungsbezeichnung im englischen (spectator genre) und im romanischen Raum (französisch: ,les spectateurs'; spanisch: ,los espectadores'; italienisch: ,gli spettatori') wird, setzt mit der Publikation der zweiten Zeitschrift ein. Während am Titel des Tatler noch der Fokus auf die Geschwätzigkeit herauszulesen ist, verweist der Titel des Spectator bereits auf den Sehsinn und die Tätigkeit des Betrachtens, des Wahrnehmens oder des Beobachtens: ${ }^{45}$

$\mathrm{He}$ [Mr. Spectator] is thus the very locus in which, or rather through which, the subject finds its place, the agency which allows the symbolic structuring of a gaze which opens up for itself the unlimited field of desire. But being mute, he, unlike Bickerstaff, cannot intervene in the process and falsify or shortcircuit it. The mastery of spectatorial discourse is thereby transferred from the fictive locus of its origin to that of its effects on the reader [kursiv im Orig.]. (Bony 1999, 239)

Im Allgemeinen sind Mr. Spectator und alle nachfolgenden spectatorialen Verfasser*innen schweigsame Figuren, die lieber niederschreiben, was sie wahrnehmen, als sich darüber mit anderen im direkten, mündlichen Gespräch auszutauschen. Stets situieren sie sich außerhalb der erzählten Ereignisse, quasi als unbeteiligte, neugierige

auch Joseph Addison in seinen Spectator-Essays ,On the Pleasures of Imagination“ (cf. Spectator No. 411-421).

$44 \mathrm{Im}$ 18. Jahrhundert entwickelt sich das Wissensideal des Empirismus. Als Grundlage für die empiristische Welterkenntnis dienen die sinnliche Wahrnehmung und die damit verbundene induktive Methode, die von allen Menschen angewandt werden kann und womit der Zugang zum Wissen nun (zumindest theoretisch) allen offensteht (cf. Neumann 2009, 85). Empiristische Erkenntnisse über die Welt werden aus der direkten sinnlichen Wahrnehmung gewonnen, das heißt ,,auf den Tatsachen einer objektiv und mittelbar beobachtbaren Wirklichkeit, wie sie über Sinnesorgane, vor allem über den Blick, wahrnehmbar wird“ (ibid., 119). Generell postulieren die Wochenschriftenproduzent*innen ,eine direkte Entsprechung zwischen subjektiver Sinneswahrnehmung und objektiver Welt [...], welche sicherstelle, dass Subjekte - als neutrale Beobachter - die Welt erkennen, wie sie , wirklich` ist. Dementsprechend wird das, was wahrnehmbar ist, als das begriffen, was tatsächlich ,ist““ (ibid.).

$45 \mathrm{Zu}$ den unterschiedlichen Konzeptionen von Isaac Bickerstaff als geselliger, geschwätziger und Mr. Spectator als stiller, die Gesellschaft meidender Charakter siehe Alain Bony (1999, 238-240; 265-266), für den Mr. Spectator mehr einen ,point of view“ als einen „,narrator“ darstellt, wobei „his muteness establishes a direct link, without the deviation or intervention of conversation, between the gaze and writing [kursiv im Orig.]“ (238). 
,stumme ' Zeug*innen, die nicht in die Ereignisse eingreifen. ${ }^{46}$ Sie bleiben meist (relativ) neutrale Figuren, die auch nicht von außen beschrieben werden und somit unfassbar bleiben. Diese neuen modernen Held*innenfiguren vom Typ eines Mr. Spectator, die den Raum um sich herum auf voyeuristische Weise wahrnehmen, stehen folglich im Gegensatz zu den Held*innen der pikaresken Tradition. Ihre Plausibilität beziehungsweise Stimmigkeit erhalten die Verfassungsinstanzen erst durch ihre Erzählungen (cf. Bony 1999, 239; Pucci 2001, 20-21).

Der spectatoriale Blick eines Mr. Spectator ist direkt verbunden mit dem Raum und der Raumwahrnehmung, genauso wie die Redseligkeit eines Isaac Bickerstaff im Tatler mit der (Konversations-)Zeit in Verbindung steht (cf. Bony 1999, 265). Im Zusammenhang mit der Wahrnehmung des umgebenden Raumes steht das Niederschreiben des Wahrgenommenen im (Text-)Raum der Zeitschrift. Diese doppelte Räumlichkeit im Spectator ist für Bony ein besonderes Merkmal: ,[T] he spatiality of the world where the spectatorial field of vision opens up, [and] the spatiality of the written word where it ,prints' itself and opens itself up to the gaze of its counterpart, the readertraveller wandering through textual space“" (ibid., 266).

Durch die Randständigkeit der Verfassungsinstanzen öffnet sich das spectatoriale Blickfeld zudem von der Peripherie auf die Welt. Aus der marginalen Position der Peripherie ,zeichnen' die Verfassungsinstanzen Porträts unterschiedlicher sozialer Typen in öffentlichen wie in privaten Räumen und stellen dem Publikum somit neue ästhetische, ethische und epistemologische Modelle für die im Wandel begriffene Gesellschaft vor (cf. Pucci 2001, 19). ${ }^{47}$ Über die Rezeptionstätigkeit sollen die besonderen visuellen Fähigkeiten der Verfasser*innen ,[...] auch die Sehfähigkeit [der Leser*innen] schärfen und [ihnen], ganz im Sinne der den Wochenschriften gemeinsamen Besserungsintention, den Blick für die verborgenen Triebfedern sowie die Vielgestaltigkeit des Menschen öffnen“" (Graeber 1986, 50).

Neben der Fremdbeobachtung spielt die Selbstbeobachtung in der Spectator-Gattung eine wichtige Rolle, denn Menschenkenntnis schließt Selbstkenntnis ein. Den Blick auf die eigene Identität werfen die Verfasser*innen vor allem dann, wenn sie sich mit ihrem eigenen Charakter auseinandersetzen und diesen betonen, wobei der Schwerpunkt auf dem Selbstverständnis liegt, mit dem insbesondere die männlichen

46 Der aus dem Lateinischen von ,spectare‘ (zusehen, etwas betrachten) stammende Begriff ,spectator' verweist bereits auf den räumlich getrennten Ort, von dem aus ein/e Zuschauer*in ein Schauspiel (spectacle) beobachtet. Mit der Aufwertung der Praktiken des Blicks und des Beurteilens rückt die abseits der zentralen Bühne sitzende zuschauende Person ins Zentrum der Aufmerksamkeit. In der Aufklärung ist es zudem üblich, solch scheinbar ,neutrale“ Begriffe (wie den des ,spectator') zu verwenden, um die Geschlechterfrage durch die Idealisierung einer unparteiischen Neutralität zu beseitigen, auch wenn sich der ,spectator'-Begriff fast durchwegs auf Figuren bezieht, die in den Wochenschriften als männlich identifiziert werden (cf. Pucci 2001, 6).

47 Zur Bedeutung des Charakterbildes als spezifischer ,Parameter der Orientierung ‘ im Zuge des Aufbaus einer protestantisch-bürgerlichen Gesellschaft in Van Effens Misantrope siehe Ertlers (2012b) Artikel zum „Charakterbild in den Moralischen Wochenschriften“, in dem er die Porträts von bürgerlichen Idealtypen als ,Gradmesser' für die neue funktional differenzierte Gesellschaft deutet. 
Verfasser die Selbstbeobachtung vornehmen. Dieses Selbstverständnis erlaubt Mr. Spectator und den nachkommenden Verfasser*innen schließlich, die Tätigkeit von gebildeten, reflektierten Beobachter*innen der Gesellschaft ihrer Zeit auszuüben. In Van Effens Nouveau Spectateur (1723-1725) erlangt der Verfasser „,[d]ie Befähigung zum Menschenbeobachter [...] vor allem durch drei Elemente: Reisen, Erziehung und analytische Fähigkeiten (vgl. NS1 I, 14)“ (Fischer 2014, 192). ${ }^{48}$

Woher kommt das Interesse an der Fremd- und Selbstbeobachtung? Dieser veränderte Fokus hängt mit dem generellen Wandel der Perspektive von Gott auf den Menschen, von einem theozentrischen auf ein anthropozentrisches Weltbild zusammen, das mit dem Beginn der Neuzeit einsetzt. Der Mensch hört auf, die Welt nur mehr über Gott und das Göttliche zu erklären und beginnt zu erforschen, wie das Individuum mit der Welt zusammenhängt. Es wird begonnen, den Menschen in den Mittelpunkt der Überlegungen zu stellen und ihn zu beobachten, sich für seinen Körper, seine Verhaltensweisen, seine Tugenden, Laster etc. zu interessieren. Da fortan nicht mehr alles als gottgegeben hingenommen werden kann, wird versucht andere, ,natürliche` Erklärungen für die menschliche Existenz zu finden.

Die Thematisierung eines subjektiven Ichs, das in der Renaissancemalerei in der Form von Fremd- und Selbstporträts aufkommt, ${ }^{49}$ findet immer mehr Eingang in wissenschaftliche Diskussionen und literarische Werke (cf. Hirschfelder 2017, 363). ${ }^{50}$ Die Fragen ,Was ist das Ich?‘ oder ,Welche Form hat das Ich? ${ }^{\text {51 }}$ werden zu zentralen Fragen der Autor*innen der Klassik und die Suche nach möglichen Antworten und Erklärungen führt sie geradewegs in die Anthropologie, womit im 17. Jahrhundert (und bis ins 19. Jahrhundert) die Anatomie gemeint ist und in deren Mittelpunkt der Begriff des

48 Innerhalb des digitalen Wochenschriften-Repositoriums werden Selbst- und Fremdbeschreibungen der unterschiedlichsten Verfassungsinstanzen zuweilen unter den ErzählformMarkups Selbstporträt und Fremdporträt angezeigt.

49 Im 15. und 16. Jahrhundert wächst die Zahl von bildkünstlerischen Porträtdarstellungen stark an. Zur formelhaften Darstellung von Heiligen sowie von Gestalten aus Mythologie und Geschichte (z. B. in Form von Herrscher*innenporträts) gesellt sich die realistische Darstellung von Einzelpersonen, die das Individuum in seiner charakterlich-körperlichen Disposition festhalten. Zum historischen Prozess der Individualisierung im Spiegel der Porträtmalerei siehe Christoph Wagner (2001).

50 So zum Beispiel wendet man sich im 17. Jahrhundert in den Naturwissenschaften immer mehr der menschlichen Anatomie (durch Sektionen) zu, deren Wissensstand seit Galenus (129-ca. 199 n. Chr.) unverändert geblieben war. Für die französische Moralistik der zweiten Jahrhunderthälfte wird die Anatomie zum Forschungsinstrument, um die Regungen im Inneren des Menschen zu analysieren (cf. Van Delft 2005a). In der Literatur erstarkt im Zeitalter der Aufklärung das Interesse an autobiografischen Werken, in denen der Mensch und sein Verhalten, Handeln und Leiden im Mittelpunkt stehen. Zur Entwicklung der Autobiografie und Selbstdarstellung im Europa des 18. Jahrhunderts siehe Ralph-Rainer Wuthenow (1974) Das erinnerte Ich.

51 Diese Frage wird von Blaise Pascal (1623-1663) in seinen Pensées (1670) derart formuliert, da für ihn das Ich eine Form darstellt (cf. Van Delft 2005a, 11). 
Charakters steht. ${ }^{52}$ Louis van Delft (2005a, 9-18) erklärt die folgenden zwei Ideenpole, die sich auf der Suche nach dem Ich (beziehungsweise nach dem menschlichen Charakter, der menschlichen Natur - heute wäre ,Identität‘ die treffende Bezeichnung) herauskristallisieren: Der erste Lösungs- oder Antwortvorschlag greift auf Theophrasts (ca. 372-ca. 287 v. Chr.) Charakterlehre (und das seit der Antike bestehende Viererschema $)^{53}$ zurück und begreift das Ich als eine homogene und invariante, weil essenzialistische (das heißt, von Natur aus' fixierte) Form. In der Literatur verwenden die klassischen Moralist*innen die literarische Form des Charakters, um solche Ich-Formen zu beschreiben, wobei ,[s]owohl der Charakter als anthropologischer Begriff als auch der Charakter als literarisches Genre [...] eine klassifizierende Analyse voraus[setzt] [kursiv im Orig.]" (ibid., 16) und danach strebt, die menschliche Natur in eine Form beziehungsweise Ordnung zu bringen. Die „Weise, in der [d]ie [Analyse] das Wirkliche erfasst, interpretiert und in Ordnung - in Form - bringt, ist die Lokalisierung“ (ibid.). Die Verortung von Charakteren führt zu einem topologischen Diskurs mit eigener Semiotik (vor allem aus dem Bereich der Physiognomie), der sich in Form von topografischen, anatomischen Atlanten wie der Steirischen Völkertafel ${ }^{54}$ manifestiert.

52 Die Hinwendung zum Charakter ist auch im aufklärerischen Geschlechterdiskurs anzutreffen, und zwar zu der Zeit, als das neuzeitliche Bezugssystem des Standes, das die Unterschiede von Frauen und Männern in der Gesellschaft definierte, auszudienen beginnt und das Bezugssystem des biologisch begründeten Geschlechtscharakters an seine Stelle tritt (cf. Hausen 1976).

53 Das Viererschema geht auf den griechischen Philosophen Empedokles (ca. 495 bis ca. 435 v. Chr.) zurück, der mit seiner naturphilosophischen Vier-Elemente-Lehre die Entstehung des Kosmos und des Menschen auf das Verhältnis von Feuer, Wasser, Luft und Erde zurückführt. Die Vierzahl fungiert bis etwa 1800 als bestimmende Größe aller Ordnungsentwürfe und umfasst z. B. das Jahr, den Lebenszyklus, die Kardinalfarben und die Himmelsrichtungen. ,[I]n der Doktrin der vier Säfte (humores, daher: Humoralpathologie)“ bestimmt das „Viererschema [...] seit Hippokrates auch das medizinische Denken“ (Böhme 1997, 30). Ebenso wird ,die historisch folgenreiche Geschlechterpolarität von Mann/Feuer (trocken/ warm) und Frau/Wasser (feucht/kalt)“ (ibid., 31) im 4. Jahrhundert v. Chr. in das medizinische Denken eingeführt. Zu den vier Elementen im Zusammenhang mit der Anthropologie siehe Hartmut Böhme (1997).

54 Die Europäische Völkertafel ist eine der ersten schematisch-komparativen Darstellungen oder „synoptischen Übersichten“ (Van Delft 2005a, 11) über die stereotypen Nationenbilder. Bei der Völkertafel handelt es sich um ein $104 \mathrm{~cm}$ x $126 \mathrm{~cm}$ großes Ölgemälde aus dem ersten Drittel des 18. Jahrhunderts, das aufgrund seines Herstellungsortes in Bad Aussee in der Steiermark fälschlicherweise auch „Steirische Völkertafel“ genannt wird. Inhaltlich ist sie aber mit dem Augsburger Kupferstich von Friedrich Leopold ident, und es wird angenommen, dass sie auf diesen zurückgeht. Auf der Völkertafel sind zehn Kategorien ethnografischer Natur abgebildet, anhand derer die Völker unterschieden und charakterisiert werden. Zum einen finden sich darauf landeskundliche Kategorien (historische, politische, wirtschaftliche Fakten), zum anderen Charakterbeschreibungen (cf. Stanzel 1998, 13-18). Die Völkertafel wurde mehrfach repliziert, wobei Stanzel von drei Tafeln in öffentlichen Museen und vier weiteren in Privatbesitz ausgeht (cf. id. 1999, 28). 
Im Gegensatz dazu begreift der zweite Lösungsvorschlag auf der Suche nach dem Ich das Ich (den menschlichen Charakter, die menschliche Natur) als instabile, sich verändernde Form, was am besten in den Essais (1580-1588) von Michel de Montaigne (1533-1592), dem wichtigsten Vertreter dieser Auffassung, zum Ausdruck kommt. Durch die Methode der Selbstbeobachtung hat er in seinen Essais gezeigt, dass die Erkundung des Ich nie abgeschlossen ist. Durch seine wandelbare Form hat der Mensch somit auch die Möglichkeit, sein Ich, seinen Charakter und sein Verhalten zu verändern, was eine grundlegende Beobachtung mit Bezug auf die proklamierte Besserungsintention der Moralischen Wochenschriften darstellt. Denn würden die spectatorialen Produzent*innen von einem statischen Ich ausgehen, wäre der belehrende Aspekt des ,prodesse“ darin hinfällig. In der Essay-Form (die ebenfalls in den einzelnen Nummern der moralischen Periodika vorkommt) als Ausdruck der Unabgeschlossenheit spiegelt sich zudem die Bewegung wider, mit der das Ich erkundet wird. Als literarische Ausdrucksform eignet sich neben dem Essay die Maxime, denn ,[f]ür sich genommen bietet eine Maxime immer nur eine partielle, unvollständige, nicht beendete, nicht vollendete Beschreibung der Form des Ich. Sie ist strukturell und semantisch autonom und sich selbst genügend“ (Van Delft 2005a, 15). Die analytische Vorgangsweise folgt dem Modell der Anatomie: Wie beim Sezieren eines Organismus arbeitet sich der/die Moralist*in Stück für Stück ins Innere des Menschen vor, zum Kern des Ich: ${ }^{55}$ „Von Montaigne bis Marivaux (und sogar noch darüber hinaus) hat dieses Modell den Blick der Beobachter bestimmt, die die Form des Ich genauer ,belauern' (wie es in den Essais heißt), indem sie bis zu den innersten und geheimsten Schichten , vordringen“ wollen [kursiv im Orig.]“ (ibid., 16-17).

\subsection{ZUSAMMENFASSUNG}

Alle in diesem Abschnitt genannten Gattungsmerkmale (i. e. periodische Erscheinung, Übersetzungen/Nachahmungen/Adaptationen, weibliches Lesepublikum, fiktive Produzent*innen, Soziabilität, vielfältige literarische Formen und Gattungen sowie Menschenbeobachtung) sind in den französisch- und spanischsprachigen Moralischen Wochenschriften in unterschiedlichen Ausmaßen anzutreffen. Im Zuge der Wissens- und Welterzeugung des neu entstehenden bürgerlichen Lesepublikums spielt vor allem die Menschenbeobachtung eine eminente Rolle, zielen die Produzent*innen doch auf die (moralische) Besserung ihrer Leser*innenschaft ab. Dazu genügt es allerdings nicht, die ideale Welt mit ihren erwünschten (geschlechtsspezifischen) Werten, Normen und Praktiken nur zu präsentieren, denn das Publikum muss erst darin geschult werden, die Unterschiede zwischen dem gegenwärtigen Ist-Zustand und dem angestrebten SollZustand wahrzunehmen, um in weiterer Folge sich selbst und Andere in diesen SollZustand zu versetzen.

Primär muss auch noch die Neugier des Publikums, die Montaigne als Bestandteil der widersprüchlichen menschlichen Natur (und somit als anthropologische Konstan-

55 Zur Entwicklung des Fokus auf das Beobachten der Sitten, das stark mit dem anthropologischen/anatomischen Beobachten des Körpers einhergeht, siehe Louis van Delft (2005b) Les spectateurs de la vie. Généalogie du regard moraliste. 